\title{
Medición intraoperatoria con una aplicación para teléfono inteligente que mejora la precisión de las osteotomías desrotadoras
}

\author{
Andrés Ferreyra," Ramiro Olleac," J. Javier Masquijo" \\ "Departamento de Ortopedia y Traumatología Infantil, Sanatorio Allende, Córdoba, Argentina \\ *"Servicio de Ortopedia Infantil, Hospital de Clínicas "Presidente Dr. Nicolás Avellaneda", San Miguel de Tucumán, Argentina
}

\begin{abstract}
RESUMEN
El desarrollo de teléfonos inteligentes ha creado nuevas oportunidades para incorporar la tecnología médica en la práctica clínica diaria. La medición intraoperatoria exacta de los grados necesarios de corrección es un desafío frecuente para el cirujano cuando realiza osteotomías desrotadoras. Por lo general, se utilizan clavijas divergentes colocadas proximal y distal a la osteotomía que, luego de la desrotación, deben quedar paralelas. Sin embargo, la medición de estos grados, en general, se hace por estimación visual, lo que suele ser poco preciso. El objetivo de este estudio es describir un detalle técnico que combina la aplicación de clavijas divergentes con la medición intraoperatoria mediante telefonía móvil para mejorar la precisión de las osteotomías desrotadoras.
\end{abstract}

Palabras clave: Osteotomía desrotadora; técnica quirúrgica; medición intraoperatoria; teléfono inteligente; aplicaciones.

Nivel de Evidencia: $\mathrm{V}$

Intraoperative Measurement with a Smartphone App That Improves the Accuracy of Derotational Osteotomies

\section{ABSTRACT}

Smartphone technology has created new opportunities to incorporate medical technology into daily clinical practice. Accurate intraoperative measurement of the desired derotation angle is a frequent challenge for the surgeon when performing derotational osteotomies. Divergent pins are commonly used proximal and distal to the osteotomy, which after derotation should remain parallel. However, the measurement of the derotation angle is usually performed by visual estimation, which could be unreliable. The aim of this study is to describe a technical detail that combines the application of divergent pins with intraoperative measurement by mobile phone to improve the accuracy of derotational osteotomies.

Key words: Derotational osteotomy; surgical technique; intraoperative measurement; smartphone; apps.

Level of Evidence: $\mathrm{V}$

\section{INTRODUCCIÓN}

El desarrollo de teléfonos inteligentes ha creado nuevas oportunidades para incorporar la tecnología médica en la práctica clínica diaria. ${ }^{1,2}$ En 2015, alrededor del $90 \%$ de los médicos del Reino Unido utilizaba este tipo de dispositivos para la evaluación o el tratamiento de sus pacientes. ${ }^{2}$ Los teléfonos inteligentes presentan un rol innovador para la especialidad de traumatología. ${ }^{3}$ El crecimiento y desarrollo rápidos de diversas aplicaciones en los últimos años ha llevado a que se realicen numerosas investigaciones para evaluar su efectividad y beneficio clínico. ${ }^{2} \mathrm{La}$ incorporación de inclinómetros y sensores de aceleración en los teléfonos inteligentes permitió expandir su uso como goniómetros digitales para medir el rango de movilidad del codo, ${ }^{4}$ la rodilla, ${ }^{5}$ la deformidad rotacional del fémur, ${ }^{6}$ la rotación del tronco en pacientes con escoliosis ${ }^{7,8}$ y la articulación metatarsofalángica del primer rayo, ${ }^{9}$ entre otros. 
Existen múltiples cuadros que requieren osteotomías desrotadoras. Entre los más frecuentes se encuentran la parálisis braquial obstétrica, la disfunción del brazo de palanca en pacientes con enfermedades neuromusculares y dolor o inestabilidad rotulofemoral asociados a deformidad en el plano axial. La medición intraoperatoria exacta de los grados necesarios de corrección es un desafío frecuente para el cirujano cuando realiza estas osteotomías. Algunos autores utilizan clavijas divergentes colocadas proximal y distal a la osteotomía que, luego de la desrotación, deben quedar paralelas. Sin embargo, la medición de estos grados, en general, se hace por estimación visual, lo que suele ser poco preciso. ${ }^{10,11}$ El objetivo de este estudio es describir un detalle técnico que combina la aplicación de clavijas divergentes con la medición intraoperatoria mediante telefonía móvil para mejorar la precisión de las osteotomías desrotadoras.
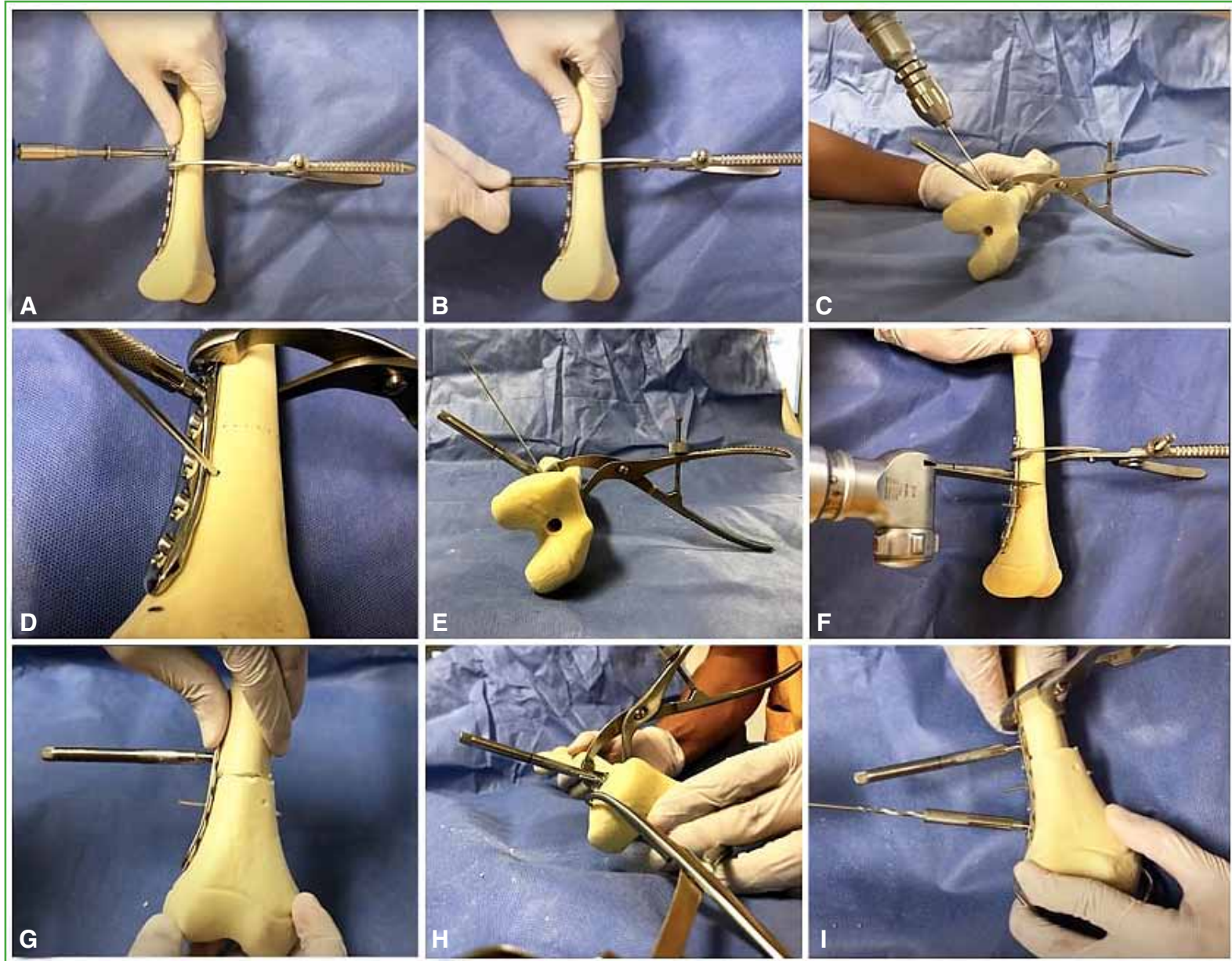

Figura. Simulación de la técnica con hueso plástico de fémur. A. Fijación de la placa al hueso. B. Colocación de la guía de bloqueo para utilizar de referencia. C. Clavija divergente, formando un ángulo con la guía previa. D. La clavija debe estar adyacente a un orificio de la placa, distal al sitio de la osteotomía. E. Disposición óptima para realizar la medición con la aplicación para teléfono inteligente. F. Osteotomía planificada. G. Desrotación del fragmento distal del fémur, haciendo coincidir la clavija con el orificio correspondiente de la placa. H. Comprobación de que la clavija y la guía están paralelas. I. Fijación de la placa con los tornillos restantes. 


\section{Descripción de la técnica}

En el video que acompaña este artículo, se analiza, con detalles, el paso a paso de la técnica empleada (Video).

Se realiza el abordaje quirúrgico pertinente de acuerdo con el segmento afectado, se avanza hasta identificar el sitio de osteotomía, se eleva el periostio en forma prolija para que luego permita su cierre. Se coloca una placa LCDCP del tamaño adecuado. Se fija la placa con el tornillo correspondiente en uno de los orificios proximales al sitio de la osteotomía (Figura A). Se coloca la guía para tornillos bloqueados en el orificio proximal más cercano a la futura osteotomía (Figura B). A continuación, se coloca una clavija en forma divergente a la guía previa (Figura C), con una angulación aproximada a la calculada antes de la cirugía. Debe ingresar a la altura de uno de los orificios distales a la osteotomía (Figura D). Para medir los grados de corrección, se utiliza una aplicación para teléfono inteligente (Navi-gator, en desarrollo). Un asistente toma la fotografía necesaria para medir el ángulo entre la clavija y la guía de bloqueo. Esto puede hacerse colocando el teléfono cuidadosamente en una bolsa plástica estéril o directamente desde fuera del campo quirúrgico (Figura E). Si no es el ángulo planificado: retirar la clavija, colocar nuevamente en otra posición y repetir la medición. Una vez obtenido el ángulo deseado, se corta la clavija a 2-3 $\mathrm{cm}$ del hueso. Se realiza la osteotomía con mecha y escoplo o sierra oscilante (Figura F). Una vez completada la osteotomía, se efectúa la desrotación trasladando el fragmento distal para hacer coincidir la clavija con el orificio correspondiente (Figura G). Se comprueba que la guía de bloqueo y la clavija estén paralelas (Figura H), se fija la placa en la posición deseada con una pinza davier y se colocan los tornillos restantes de la placa (Figura I). Luego se procede al cierre por planos.

\section{DISCUSIÓN}

Las osteotomías desrotadoras son un procedimiento quirúrgico frecuente para tratar diversas enfermedades ortopédicas. El grado de desrotación necesario suele ser evaluado por el cirujano, durante la cirugía, de manera aproximada, mediante una estimación visual. Para evaluar el grado de corrección, algunos cirujanos utilizan clavijas divergentes, marcas realizadas con sierra en el hueso afectado o evaluando el rango de movilidad pasiva bajo anestesia. ${ }^{12}$ Kozin propone usar una clavija adyacente al orificio de la placa distal a la osteotomía que, luego de la desrotación, quedaría alineada con la placa al ingresar en el orificio correspondiente. ${ }^{13} \mathrm{Si}$ bien este gesto es de gran utilidad para mantener alineado el miembro hasta realizar la fijación definitiva, el autor no describe el método de medición del ángulo por corregir. En este artículo, comunicamos el empleo de una aplicación para teléfonos móviles que permite medir el ángulo formado entre la clavija y la guía de bloqueo de la placa. Esto facilita una evaluación precisa del ángulo en el plano axial por corregir.

Existen escasos reportes sobre la aplicación de la telefonía inteligente durante la cirugía. Peters y cols. ${ }^{14}$ evaluaron la orientación del componente acetabular de las prótesis de cadera mediante una aplicación de goniometría colocando el teléfono inteligente dentro de una bolsa estéril durante la cirugía. Graham y cols. ${ }^{15}$ compararon las mediciones entre dos clavijas divergentes colocadas en huesos de plástico con tres mediciones: por estimación visual, con guías metálicas estáticas con $10^{\circ}$ de diferencia progresiva y con el goniómetro digital de un teléfono inteligente, y utilizaron como valor real las mediciones obtenidas por tomografía. De esta manera, demostraron que las mediciones angulares con teléfonos inteligentes mejoran la exactitud y la precisión, comparadas con las de las técnicas convencionales.

El uso de aplicaciones para la medición de ángulos disponibles para teléfonos inteligentes permitiría mejorar la exactitud de la desrotación necesaria para los pacientes con deformidades rotacionales de los miembros. Se requieren estudios clínicos que comparen la técnica tradicional mediante estimación visual con la propuesta en esta nota técnica para validar su utilidad.

Conflicto de intereses: Los autores no declaran conflictos de intereses. La aplicación de teléfono inteligente Navi-gator para Iphone utilizada en este estudio es una versión en desarrollo, propiedad de R.O. No está disponible para uso general y solo fue otorgada gratuitamente a los responsables de las pruebas de campo (JM y AF). 


\section{BIBLIOGRAFÍA}

1. Franko OI. Smartphone apps for orthopaedic surgeons. Clin Orthop Relat Res 2011;469:2042-8. https://doi.org/10.1007/s11999-011-1904-0

2. Mobasheri MH, King D, Johnston M, Gautama S, Purkayastha S, Darzi A. The ownership and clinical use of smartphones by doctors and nurses in the UK: a multicentre survey study. BMJ Innov 2015;1(4):174-81. https://doi.org/10.1136/ bmjinnov-2015-000062

3. Al-Hadithy N, Gikas PD, Al-Nammari SS. Smartphones in orthopaedics. Int Orthop 2012;36(8):1543-7. https:// doi.org/10.1007/s00264-012-1527-4

4. Behnoush B, Tavakoli N, Bazmi E, Nateghi Fard F, Pourgharib Shahi MH, Okazi A, et al. Smartphone and universal goniometer for measurement of elbow joint motions: a comparative study. Asian J Sports Med 2016;7(2):e30668. https://doi. org/10.5812/asjsm.30668

5. Ferriero G, Vercelli S, Sartorio F, Muñoz Lasa S, Ilieva E, Brigatti E, et al. Reliability of a smartphone-based goniometer for knee joint goniometry. Int J Rehabil Res 2013;36(2):146-51. https://doi.org/10.1097/ MRR.0b013e32835b8269

6. Shen YF, Huang JH, Li XL, Gao H. Evaluation of the smartphone for measurement of femoral rotational deformity. ANZ J Surg 2019;(89):E422-E427. https://doi.org/10.1111/ans.15384

7. Franko OI, Bray C, Newton PO. Validation of a scoliometer smartphone App to assess scoliosis. Pediatr Orthop 2012;32:e72-e75. https://doi.org/10.1097/BPO.0b013e31826bb109

8. Balg F, Juteau M, Theoret C, Svotelis A, Grenier G. Validity and reliability of the iPhone to measure rib hump in scoliosis. J Pediatr Orthop 2014;34:774-9. https://doi.org/10.1097/BPO.0000000000000195

9. Otter SJ, Agalliu B, Baer N, Hales G, Harvey K, James K, et al. Reliability of a smartphone goniometer application compared with traditional goniometer for measuring first metatarsophalangeal joint dorsiflexion. J Foot Ankle Res 2015;8:30. https://doi.org/10.1186/s13047-015- 0088-3

10. Delgado ED, Schoenecker PL, Rich MM, Capelli AM. Treatment of severe torsional malalignment syndrome. $J$ Pediatr Orthop 1996;16(4):484-8. https://doi.org/10.1097/00004694-199607000-00012

11. Kolp D, Ziebarth K, Slongo T. [Rotation or derotation osteotomy of the tibia]. Oper Orthop Traumatol 2017;29(2):163-72. [En alemán] https://doi.org/10.1007/s00064-016-0473-5

12. Bae DS, Waters PM. External rotation humeral osteotomy for brachial plexus birth palsy. Tech Hand Up Extrem Surg 2007;11(1):8-14. https://doi.org/10.1097/01.bth.0000248359.14448.e6

13. Kozin SH. Medial approach for humeral rotational osteotomy in children with residual brachial plexus birth palsy. Oper Tech Orthop 2007;17:88-93. https://doi.org/10.1053/j.oto.2007.01.006

14. Peters FM, Gree R, Goldstein N, Frey CT. Improving acetabular cup orientation in total hip arthroplasty by using smartphone technology. J Arthroplasty 2012;27(7):1324-30. https://doi.org/10.1016/j.arth.2011.11.014

15. Graham D, Suzuki A, Reitz C, Saxena A, Kuo J, Tetsworth K. Measurement of rotational deformity: using a smartphone application is more accurate than conventional methods. ANZ J Surg 2013;83:937-41.

https://doi.org/10.1111/ans.12371 